Check for updates

Cite this: RSC Adv., 2019, 9, 26037

\title{
Self-formed silver nanoparticles on freestanding silicon nanowire arrays featuring SERS performances $\dagger$
}

\author{
Pin-Ju Chien, ${ }^{a}$ Yongcun Zhou, ${ }^{b}$ Kun-Hung Tsai, ${ }^{a}$ Hong Phan Duong $\mathbb{D D}^{\mathrm{c}}$ \\ and Chia-Yun Chen (D) *ad
}

\begin{abstract}
Herein, the universal luminescence characteristics of porous Si nanowire arrays were exploited using a wide range of doping types and concentrations; we found that the dual-band photoluminescence intensities were correlated with the formation rates of Si nanowires with porous features; however, these intensities exhibited no evident dependence on the doping conditions. Furthermore, we demonstrated a facile and reliable transfer method implementing the freestanding Si nanowire arrays while maintaining the robust photoluminescence behaviors under bending conditions. The fabrication protocol, involving lateral etching locally at the nanowire ends, enabled the controlled formation of uniform and large-area transferred nanowires with vertical regularity. Without the additional deposition of Ag nanoparticles, these transferred Si nanowire films inherently possessed SERS sensing capability with a relative enhancement factor over 1.8 times that of the Si nanowires with electroless-deposited Ag nanoparticles, which could practically emerge as a functional design for the integration of practical biochip devices.
\end{abstract}

Received 2nd May 2019

Accepted 5th August 2019

DOI: $10.1039 /$ c9ra03273h

rsc.li/rsc-advances
SERS, which has been considered as an ultrasensitive strategy for the extraction of molecular information from complex biological environments and can even enable the detection capability of up to the single-molecule level. ${ }^{11}$

On the basis of strong localized surface plasmon resonance (LSPR) excitation, the electromagnetic field near the surfaces of noble metal nanoparticles is significantly enhanced, offering more intense Raman scattering from the detected molecules. However, the sensing performance highly relies on the spatial position of metal nanoparticles, where the particle size, shape and arrangement should be maintained for providing a reproducible and reliable SERS effect. ${ }^{12}$ In this regard, the freestanding SiNW arrays could act as promising host materials, which enabled the stabilization of dispersed metal nanoparticles on SiNW sidewalls against aggregation that could significantly degrade their sensing performance. Essentially, an additional deposition process, such as electroless plating, ${ }^{\mathbf{1 3}}$ drop coating, ${ }^{14}$ physical deposition and hydrothermal growth, ${ }^{15-17}$ for coating metal nanoparticles on SiNWs is required, which may be time consuming and challenging to create spatially distributed features that are necessary to provide high SERS sensitivity. Overall, in this study, we demonstrated flexible SERS sensors by implementing the controlled formation of freestanding porous SiNWs decorated with self-formed Ag nanoparticles (AgNPs). To achieve this aim, we exploited a transfer technique involving lateral etching locally at the end of the SiNW arrays. Bending tests to determine the light-emitting characteristics of transferred SiNW structures were conducted to examine the reliability of the transfer 
process. We further found that these freestanding SiNW films possessed a remarkable SERS sensing performance owing to the inherent decoration of the dispersed AgNPs on SiNWs.

\section{Experimental details}

\section{Fabrication}

Single-crystalline and (100)-oriented Si substrates made by the Czochralski method with various dopant types and concentrations, including $\mathrm{n}^{+}(0.1-0.5 \mathrm{ohm} \mathrm{cm}), \mathrm{n}$ (1-10 ohm cm), p (1-10 ohm cm), $\mathrm{p}^{+}(0.1-0.5 \mathrm{ohm} \mathrm{cm})$ and $\mathrm{p}^{++}(0.01-0.05 \mathrm{ohm} \mathrm{cm})$, were utilized for the preparation of solid and porous SiNW arrays. Prior to the etching reaction, the Si substrates were carefully cleaned several times via ultrasonication with acetone, isopropyl alcohol (IPA) and deionized water (DI). After this, two distinct metal-assisted chemical etching (MaCE) processes were performed for the preparation of regular Si nanowire arrays. ${ }^{\mathbf{1 8 - 2 2}}$ In the one-step MaCE method, the Si substrates were immersed in the etching solutions containing $0.02 \mathrm{M}$ of $\mathrm{AgNO}_{3}$ and $4.6 \mathrm{M}$ of $\mathrm{HF}$ at $30^{\circ} \mathrm{C}$ for $10 \mathrm{~min}$. In the two-step MaCE method, the $\mathrm{Si}$ substrates were dipped in the mixed solution containing $0.005 \mathrm{M}$ of $\mathrm{AgNO}_{3}$ and $4.6 \mathrm{M}$ of $\mathrm{HF}$ at $25{ }^{\circ} \mathrm{C}$ for $10 \mathrm{~s}$ and subsequently transferred to another etching solution containing $0.3 \mathrm{M}$ of $\mathrm{H}_{2} \mathrm{O}_{2}$ and $4.6 \mathrm{M}$ of $\mathrm{HF}$ at $30{ }^{\circ} \mathrm{C}$ for $10 \mathrm{~min}$. After performing the etching experiments, the nanowire-based samples were rinsed with DI water and dried under a gentle flow of $\mathrm{N}_{2}$ gas. The electroless deposition of $\mathrm{Ag}$ nanoparticles on either SiNWs or bare Si substrates was conducted by immersing the samples in an electrolyte solution containing $0.005 \mathrm{M}$ of $\mathrm{AgNO}_{3}$ and $4.8 \mathrm{M}$ of $\mathrm{HF}$ at room temperature for $25 \mathrm{~s}$ and then rinsing with DI water followed by drying under a stream of $\mathrm{N}_{2}$ gas.

\section{Characterizations}

The microstructures and morphologies of the Si nanowires were characterized using a transmission electron microscope (TEM, JEOL 2100) and a scanning electron microscope (SEM, LEO 1530), respectively. The chemical compositions of the as-formed $\mathrm{Ag}$ nanoparticles were analyzed using an energy dispersive spectrometer (EDS, Oxford INCA). PL investigations were performed at room temperature using a light source of a lightemitting diode lamp (the outpower of $780 \mathrm{mV}$ ) with the center wavelength of $365 \mathrm{~nm}$. Raman measurements were performed using a Raman spectrometer (Jobin Yvon) based on the laser wavelength of $532 \mathrm{~nm}$.

\section{Results and discussion}

Herein, two types of SiNWs were prepared via distinct etching techniques: one-step MaCE and two-step MaCE process, as detailed in the experimental section. The involved reaction of the one-step MaCE process could be represented as follows: ${ }^{23}$

$$
\mathrm{Si}+6 \mathrm{HF}+4 \mathrm{Ag}^{+} \rightarrow \mathrm{H}_{2} \mathrm{SiF}_{6}+4 \mathrm{H}^{+}+4 \mathrm{Ag}
$$

The overall etching was initiated by the reduction of $\mathrm{Ag}^{+}$ions supplied from $\mathrm{AgNO}_{3}$ precursors that created the $\mathrm{Ag}$ seeds on $\mathrm{Si}$ surfaces, which could be attributed to the fact that the reduction potential of $\mathrm{Ag}^{+} / \mathrm{Ag}$ was more positive than the Fermi level of Si. Moreover, these formed Ag seeds oxidized the underlying $\mathrm{Si}$ atoms through hole injections. The etching reaction was sustained along the $\langle 100\rangle$ orientations by cycling reactions that included the removal of oxidized $\mathrm{Si}$ and reduction of the $\mathrm{Ag}^{+}$ ions from the aqueous solution, eventually leaving the vertically aligned solid SiNWs behind, as illustrated in the inset image of Fig. 1(a). The corresponding TEM image is presented in Fig. 1(b), indicating that the nanowire sidewalls remain crystalline and maintain the consistent atomic configuration with inner regions.

In addition, by employing the two-step MaCE process, straight SiNWs were created through the following electrochemical reaction: ${ }^{24,25}$

$$
\mathrm{Si}+n / 2 \mathrm{H}_{2} \mathrm{O}_{2}+6 \mathrm{HF} \rightarrow n \mathrm{H}_{2} \mathrm{O}+\mathrm{H}_{2} \mathrm{SiF}_{6}+(4-n) / 2 \mathrm{H}_{2}
$$

The etching of Si preferentially occurred along the $\langle 100\rangle$ direction, where the straight sinking of the predefined Ag seeds into the Si substrates was accomplished with the local oxidation and dissolution of $\mathrm{Si}$ in contact with the $\mathrm{Ag}$ seeds; although the vertical movement of the $\mathrm{Ag}$ seeds relative to the substrate planes dominated the etching reaction, the local dissolution of $\mathrm{Ag}$ seeds also occurred due to the lower electrochemical potential of $\mathrm{Ag}$ with respect to that of the involved $\mathrm{H}_{2} \mathrm{O}_{2}$ oxidants. This increased the number of $\mathrm{Ag}^{+}$ions that preferentially re-nucleated on the rough sidewalls of the etching pores owing to the lower energy barrier required at these defective sites. With the continuous supply of $\mathrm{H}_{2} \mathrm{O}_{2}$ molecules, it could be expected that the localized etching was initiated by these newly formed Ag nuclei in conjunction with the continuous sinking of primary Ag seeds. This accounted for the formation of porous (a)

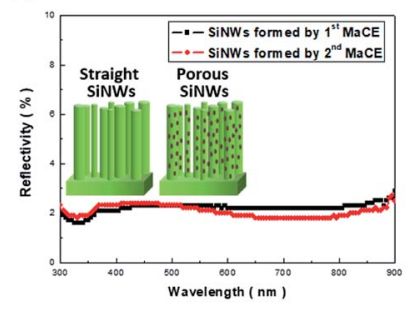

(c)

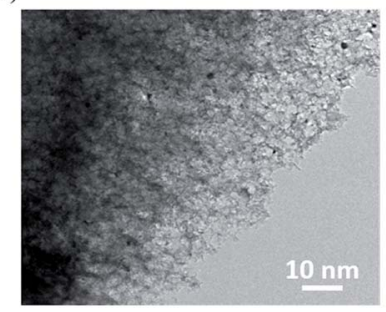

(b)

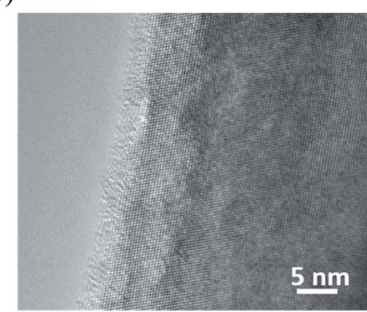

(d)

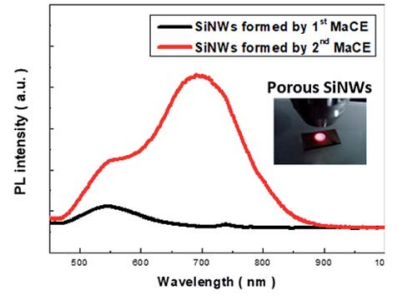

Fig. 1 (a) Light absorption spectra of both solid and porous SiNW arrays. Representative TEM images of a (b) solid SiNW and (c) porous SiNW. (d) Photoluminescence spectra of both solid and porous SiNW arrays. The inset figure shows the image of porous SiNWs illuminated with a $365 \mathrm{~nm}$ light. 
features in these one-dimensional Si nanostructures, as depicted in the inset of Fig. 1(a). These findings could be further supported by the TEM investigations, and the highly porous features on the nanowire sidewalls could be clearly observed, as evidenced in Fig. 1(c).

The corresponding light-absorption spectra of both solid and porous SiNWs are presented in Fig. 1(a), and no distinguishable features are present in the spectra of these two samples. Specifically, the average reflectivity was found to be 1.95\% (solid SiNWs) and 1.86\% (porous SiNWs). This indicated that the effect of light trapping dominated the optical absorptivity of SiNWs rather than the contributions from the nanostructure morphologies; this also implied the effective interaction of light with these two distinct SiNWs, indicating that the number of irradiated photons trapped inside these SiNWs was roughly the same. In addition, the PL spectra were compared to analyze the light-emitting properties of these SiNWs, as shown in Fig. 1(d). Contrary to the similar lightabsorption behaviours, the PL characteristics of SiNWs were found to be highly correlated with the microstructures of the tested SiNWs. The remarkable and dual PL patterns centred at both 546 and $695 \mathrm{~nm}$ could be obtained from the porous SiNWs made using the two-step MaCE process, whereas the comparatively weaker PL peak centred at $546 \mathrm{~nm}$ was found from the solid SiNWs prepared using the one-step MaCE method. Since both nanostructures could efficiently absorb the incoming light, the subsequent radiative recombination of excited charges was therefore determined by the surface structures of the light-excited SiNWs. The highly porous features appearing in the large-area skeletons of the nanowire structures could facilitate charge recombination radiatively for generating the strong red lights (center wavelength $=695 \mathrm{~nm}$ ) that could even be observed by the naked eye, as shown in the inset of Fig. 1(d). Moreover, the wideband blue/green-light emissions (center wavelength $=546 \mathrm{~nm}$ ) have been reported to originate from the rough surfaces of SiNWs, ${ }^{26,27}$ which could be observed from both the porous and solid SiNW architectures.

To exploit the universal luminescence characteristics of porous SiNWs excited by light, the two-step MaCE process was conducted under a wide range of doping conditions for $\mathrm{Si}$ substrates for the preparation of porous SiNWs with various doping levels. The comparative PL spectra reflected that the dual emission peaks of various doped SiNWs were present regardless of the doping types and concentrations, revealing that the similar light-emission mechanism was dominantly contributed by the porous nature of the formed SiNWs under a wide range of doping conditions, as presented in Fig. 2(a). Since the size and distribution of the created pores in the nanowire sidewalls were unlikely to be identical for different doped SiNWs, the effects of quantum confinement on the sizedependent emission characteristics were less validated. Instead, the PL intensities of the predominant red-light emission were dramatically different, where the porous SiNWs with slight pdoping (denoted as p SiNWs) presented the highest emission intensity, which was above 3.9 times higher than that of the heavily p-doped SiNWs (denoted as $\mathrm{p}^{++}$SiNWs).
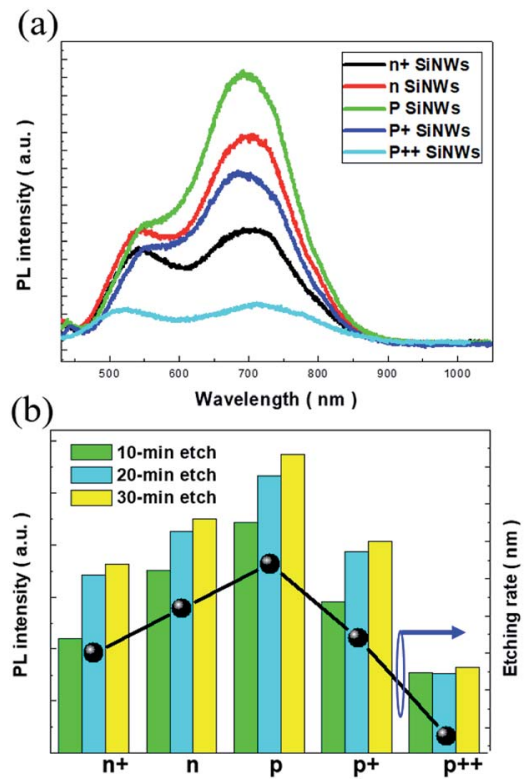

Fig. 2 (a) Photoluminescence spectra of porous SiNW arrays under various doping conditions, including $\mathrm{n}^{+}$(moderately $\mathrm{n}$-doped), $\mathrm{n}$ (lightly $n$-doped), $p$ (lightly $p$-doped), $\mathrm{p}^{+}$(moderately $\mathrm{p}$-doped) and $\mathrm{p}^{++}$(heavily $\mathrm{p}$-doped) types. (b) Comparative PL intensity at $695 \mathrm{~nm}$ of various doped SiNW arrays under three different etching durations. The corresponding etching rates for the formation of SiNW arrays were also compared.

We attributed the substantial change of emission capability to the numbers of involved pores in the Si nanocrystallites that provided effective sites for light emission. In this regard, a change in the PL intensity seemed to be correlated with the formation rate of porous SiNWs. To examine this hypothesis, a systematic validation of the doped SiNWs fabricated with various etching durations was performed, as shown in Fig. 2(b). Similar PL characteristics were presented regardless of the doping conditions and etching durations, and the universal trends in terms of PL intensity were observed: highest PL intensity was obtained from the slightly p-doped SiNWs, followed by the slightly n-doped SiNWs (n SiNWs), moderately pdoped SiNWs $\left(\mathrm{p}^{+}\right.$SiNWs), moderately $\mathrm{n}$-doped SiNWs $\left(\mathrm{n}^{+}\right.$ SiNWs) and heavily p-doped SiNWs. On the other hand, from the investigations of the etching rates of various doped $\mathrm{Si}$ substrates, it could be found that in a similar doping concentration range, the p-doped SiNWs possessed slightly higher etching rate than the n-doped SiNWs owing to the fact that the excess holes existing in the $\mathrm{Si}$ crystals facilitated the oxidation reaction in the presence of $\mathrm{H}_{2} \mathrm{O}_{2} / \mathrm{HF}$ solutions. ${ }^{28}$ However, although the doping concentrations in the $\mathrm{p}$ type material reached moderately or even heavily doped levels, the accumulation of excessive holes at the $\mathrm{Ag} / \mathrm{Si}$ interfaces created a biased potential that decelerated the succeeding Si oxidation from the hole injections of $\mathrm{H}_{2} \mathrm{O}_{2}$ oxidants, thus slowing down the etching rates. Accordingly, we found that the universal dependence of PL intensity explicitly corresponded to the etching rate for the formation of porous SiNWs, as evidenced in Fig. 2(b). Moreover, this further verified that the PL intensity of various doped 
porous SiNWs made using this simple, reliable and roomtemperature-processing method could be essentially tuned by controlling the etching durations, which might pave a rational way for synthesizing light-emitting $\mathrm{Si}$ nanostructures under controlled doping conditions.

In addition to the study on the PL properties of porous SiNWs, we further attempted to vertically transfer the regular porous SiNWs onto flexible receiver substrates. This could benefit the realization of Si-based vertical devices with flexible functionality. Fig. 3 illustrates the process flow for the detachment of functional SiNWs, followed by their complete transfer to the adhesive substrates through the facile and reliable lateral etching process. After conducting the two-step MaCE process for the preparation of porous SiNWs with desired lengths, the samples were rinsed with DI water for the complete removal of residual etchants. To initiate the lateral breakage at the end of the SiNWs, two consecutive steps were conducted for the dissolution of the $\mathrm{Ag}$ seeds: the re-nucleation of $\mathrm{Ag}$ nanoparticles followed by catalytic etching along the lateral directions. These were accomplished by immersing the as-prepared SiNWs in $\mathrm{H}_{2} \mathrm{O}_{2}$ solutions $(0.3 \mathrm{M})$ for $1 \mathrm{~min}$ and subsequently transferring them to the etching solutions containing $\mathrm{HF}$ (4.8 M) and $\mathrm{H}_{2} \mathrm{O}_{2}(1 \mathrm{M})$ solutions for $1.5 \mathrm{~min}$ at room temperature. This would create cracks at the SiNW ends and underlying substrates, thus facilitating the detachment of freestanding SiNWs from the substrates.

It has been reported that the $\mathrm{Ag}$ seeds intend to be dissolved in $\mathrm{H}_{2} \mathrm{O}_{2}$ solutions due to the reduction potential difference; ${ }^{29,30}$ this results in the formation of abundant $\mathrm{Ag}^{+}$ions attached in the bottom regions of the SiNWs. When the $\mathrm{H}_{2} \mathrm{O}_{2} / \mathrm{HF}$ etchants were introduced, these concentrated $\mathrm{Ag}^{+}$ions were reduced by hole injections from the $\mathrm{H}_{2} \mathrm{O}_{2}$ oxidants and then favourably nucleated as new $\mathrm{Ag}$ nanoparticles in the proximity of SiNW ends due to the shortest diffusion distance. These newly formed $\mathrm{Ag}$ nanoparticles acted as catalysts that facilitated the lateral etching vertically towards the sidewalls of the SiNWs, thus weakening the bonding of the SiNW films with the underlying substrates. This created the end-fractured SiNW films while maintaining the structural integrity of the vertical features that

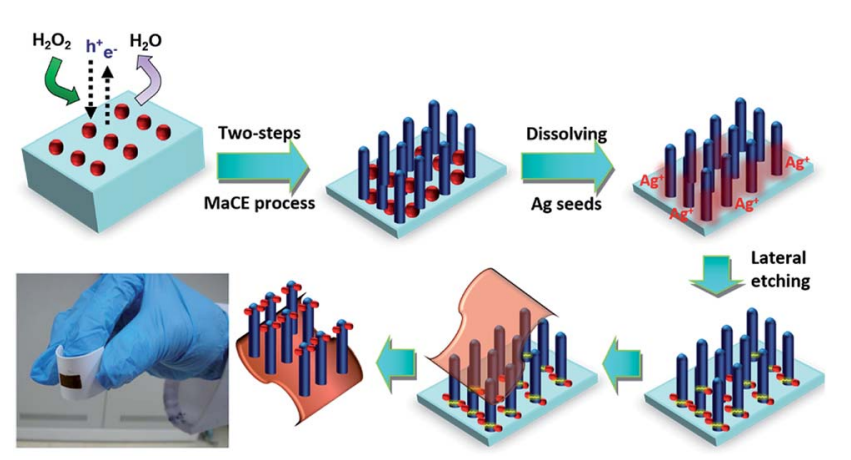

Fig. 3 Schematic process flow for the preparation of freestanding SiNW films with vertical regularity through the introduction of lateral etching locally at nanowire ends and followed by transfer to the receiver substrate. The representative image of the transferred SiNWs is also presented. could be facilely separated from the mother substrates by peeling off the attached substrates, thus successfully being transferred onto the receivers in a larger area, as shown in Fig. 3.

The detailed morphologies of the SiNWs before and after the transfer process are shown in Fig. 4, and the robust connection between the aligned porous SiNWs and Si substrates as starting materials can be observed in Fig. 4(a). By adapting the lateral etching for the creation of sidewall breakage at the SiNW ends, it horizontally broke the connection with the underlying bulk part, and thus, the freestanding SiNW films were formed, as presented in Fig. 4(b). Fig. 4(c) further demonstrates the topview comparative image showing that only the etched pits are left on the substrate surfaces after the transfer process, which indicates the uniform and large-area processing capability with no local defects or discontinuity arising from the transfer method. The cross-sectional SEM images of the transferred SiNWs films attached on the adhesive substrates are presented in Fig. 4(d), where the well-preserved alignment of the vertical configuration can be observed. To obtain an insight into the PL characteristics of the transferred SiNW films, a series of PL measurements under various bending angles were conducted, as shown in Fig. 5. The PL spectrum of flexible SiNWs was obtained for each case, i.e. a flat case, curved angles of $10^{\circ}, 20^{\circ}$ and $40^{\circ}$, and then recovery to the original flat condition. Obviously, the measured PL spectra under the abovementioned tested conditions were explicitly overlapped, highlighting the robustness and reliability of the created SiNW films.

Finally, the capability of SERS sensing based on the transferred SiNWs was exploited. Unlike the reported SERS sensors where the deposition of noble metals serving as hot spots for enhancing the Raman signals was required, ${ }^{31-33}$ these transferred SiNWs inherently constituted the hybrid architecture of AgNPs with the sizes of 5-14 nm and porous SiNW supporters. These self-formed features were attributed to the employed

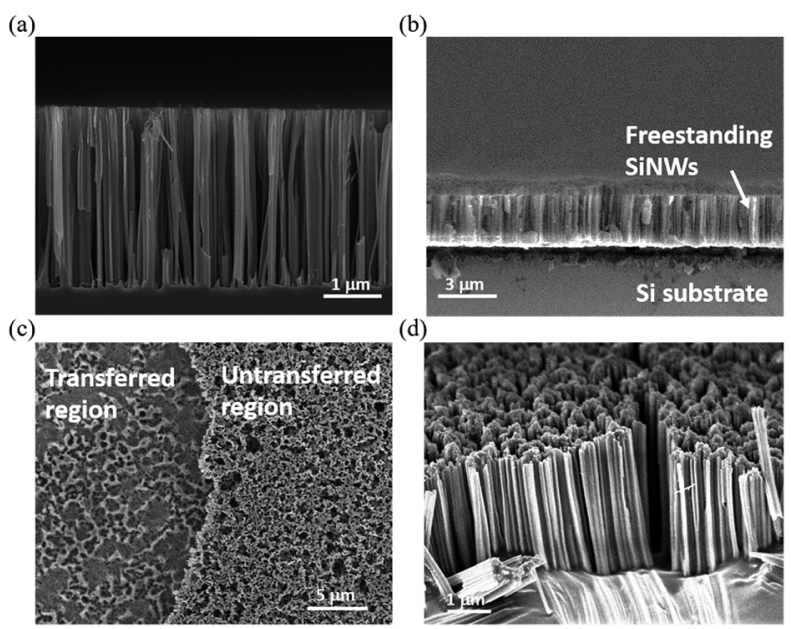

Fig. 4 Cross-sectional SEM images of the SiNW arrays (a) before and (b) after introducing the lateral etching at the SiNW ends. (c) Top-view SEM image of the mother substrate. (d) Cross-sectional SEM image of the transferred SiNW arrays. 


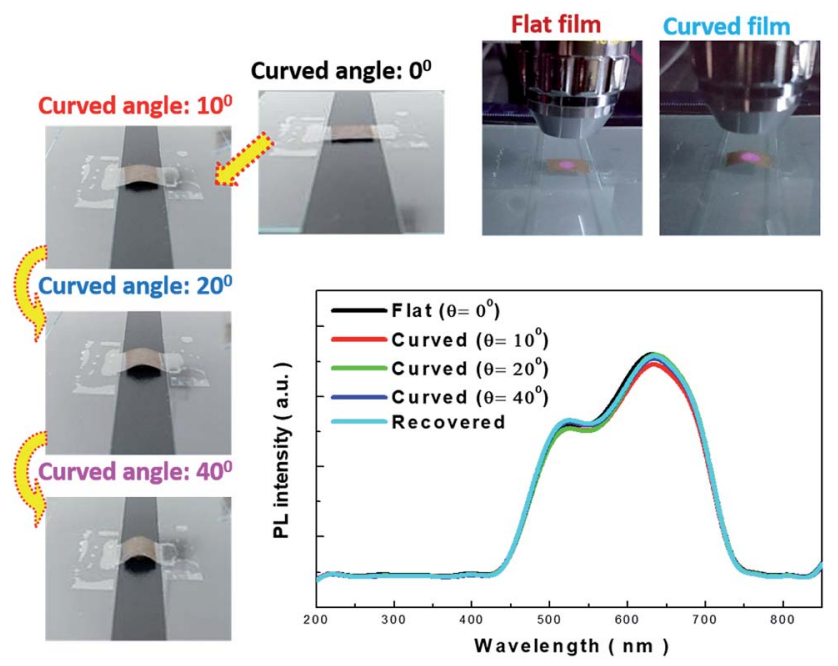

Fig. 5 Photoluminescence examinations of freestanding SiNW films under various bending angles. Each image presents the bending condition of the SiNW films for testing.

lateral etching of SiNWs that rendered the simultaneous formation of freestanding SiNW arrays and incorporated AgNPs. These were identified by the TEM investigation along with the EDS analysis of a representative freestanding SiNW, as shown in Fig. 6(a) and the ESI. $\dagger$ The SERS sensing performances were evaluated by placing the R6G dye $\left(10^{-7} \mathrm{M}\right)$ as a detection target on various sample surfaces, including the freestanding SiNW films with self-formed AgNPs, SiNWs/Si substrates and Si substrates decorated with electroless-deposited AgNPs, and bare $\mathrm{Si}$ substrates. In addition, the measurements were

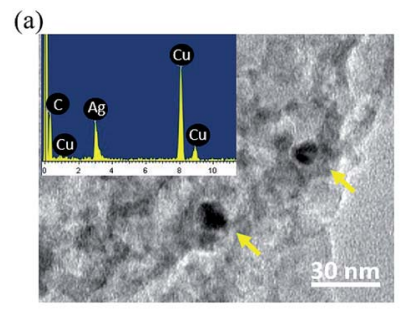

(b)

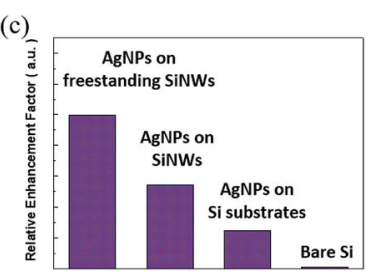

(d)

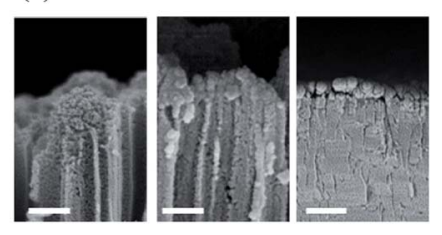

Fig. 6 (a) Representative TEM image of a transferred SiNW. The inset figure shows the corresponding EDS analysis results, indicating that the composition of the nanoparticles denoted by a yellow arrow is Ag. The signals of the $\mathrm{Cu}$ and $\mathrm{C}$ elements originated from the TEM grid. (b) Comparative Raman spectra of various SERS substrates. (c) Examination of the relative enhancement factor of various samples extracted from Raman spectra. (d) Cross-sectional SEM images of the sample surfaces including freestanding AgNPs/SiNWs (left figure), AgNPs/ SiNWs on the Si substrates (middle figure) and AgNP-loaded $\mathrm{Si}$ substrates (right figure). The scale bar is $200 \mathrm{~nm}$. conducted on the samples without bending. The corresponding Raman spectra are presented in Fig. 6(b), showing the marked Raman peaks at 1311,1511 and $1575 \mathrm{~cm}^{-1}$. This indicated that the incorporation of AgNPs into SiNWs enhanced the SERS signals than the case of the Si substrates with directly deposited AgNPs; these results could be attributed to the large surface area of SiNWs that enabled the loading of more AgNPs as active spots for the strengthening of the Raman features through the field localization of the incoming light. By contrast, the bare Si substrates presented inactive Raman interactions in the detection of R6G analytes at low-concentrations tested herein.

To further compare their sensing capabilities, the relative enhancement factors (REFs) of Raman sensing were evaluated: ${ }^{34,35}$

$$
\mathrm{REFs}=I_{\text {substrate, } \mathrm{A}} \times N_{\text {substrate, } \mathrm{A}} / I_{\text {substrate, } \mathrm{B}} \times N_{\text {substrate, } \mathrm{B}}
$$

where $I_{\text {substrate }}$ represents the characteristic SERS intensity at $1575 \mathrm{~cm}^{-1},{ }^{36}$ and $N_{\text {substrate }}$ is the number of R6G molecules on the SERS substrate. In addition, the REFs of the SiNW films with self-formed AgNPs in the presence of R6G at various concentrations are presented in the ESI. $\dagger$ The largest SERS intensity provided was found from the freestanding AgNPs/SiNWs, where the correlated REFs were above 1.8 times higher than those of the AgNPs/SiNWs on the Si substrates and approximately 4 times higher than those of the AgNP-loaded Si substrates, as presented in Fig. 6(c). These results could be elucidated by the spatial distribution of AgNPs loaded on various supporters. By examining the morphologies of the sample surfaces, it could be found that the number of deposited AgNPs on bare Si substrates was comparably less than the other two cases owing to the limited areas of planar features [Fig. 6(d)], which rendered the weakest REFs among the three samples. The freestanding SiNWs made with the transfer process had the highest REFs, with contributions from the dispersed AgNPs with small features less than $15 \mathrm{~nm}$. Thus, each AgNP could act as an active hot spot that amplified the resonant coupling of incoming light with SERS excitations. Note that these effective SERS substrates additionally released upon sophisticated processing for loading fine-size and well-distributed AgNPs; this established a rational design that might be practically integrated in the biochip platform. However, the deposition of AgNPs on SiNWs by employing the electroless plating inevitably suffered from the intended aggregation on the nanowire tips [Fig. 6(d)], and this configuration might inhibit the gathering of light excitations and therefore weaken the active electromagnetic coupling of the incident laser with Raman signals. This accounted for the comparably inferior SERS sensing activity in terms of the evaluated REFs.

\section{Conclusions}

In conclusion, systematic investigations on the PL characteristics of porous SiNW arrays were performed by varying the wide range of doping conditions. The resulting PL intensity was found to be highly dependent on the formation rates of porous features regardless of the doping types and concentrations. In 
addition, these porous Si nanowires with sound flexibility could be successfully detached from the original mother substrates using a facile and reliable transfer method while maintaining the robust PL properties under the bending conditions. Moreover, unlike the reported SERS sensors where the additional preparation of metal nanoparticles as hot spots for enhancing Raman signals was required, these transferred SiNWs inherently rendered the simultaneous formation of freestanding SiNW arrays and decorated AgNPs, thus resulting in remarkable SERS sensing performances with improved REFs over 1.8 times higher than those of the SiNWs with electroless-deposited AgNPs. This design could open new routes to the self-formed and flexible SERS sensing platform, with a potential impact on biological detection, chemical analysis and other functional applications.

\section{Conflicts of interest}

There are no conflicts of interest to declare.

\section{Acknowledgements}

This study was financially supported by the Ministry of Science and Technology of Taiwan (MOST 107-2221-E-006-013-MY3), and Hierarchical Green-Energy Materials (Hi-GEM) Research Center, from the Featured Areas Research Center Program within the framework of the Higher Education Sprout Project by the Ministry of Education (MOE) and the Ministry of Science and Technology (MOST 107-3017-F-006-003) in Taiwan. The authors greatly acknowledge the Center for Micro/Nano Science and Technology, National Cheng Kung University for the facilities provided for conducting material characterizations.

\section{Notes and references}

1 N. Koshida and H. Koyama, Jpn. J. Appl. Phys., 1991, 30, 7B. 2 Z. Y. Xu, M. Gal and M. Gross, Appl. Phys. Lett., 1992, 60, 1375.

3 L. Lin, S. Guo, X. Sun, J. Feng and Y. Wang, Nanoscale Res. Lett., 2010, 5, 1822.

4 A. I. Hochbaum, D. Gargas, Y. J. Hwang and P. Yang, Nano Lett., 2009, 9, 3550-3554.

5 Y. Qu, H. Zhou and X. Duan, Nanoscale, 2011, 3, 4060-4068.

6 Y. Qu, L. Liao, Y. Li, H. Zhang, Y. Huang and X. Duan, Nano Lett., 2009, 9, 4539-4543.

7 K. E. Plass, M. A. Filler, J. M. Spurgeon, B. M. Kayes, S. Maldonado, B. S. Brunschwig, H. A. Atwater and N. S. Lewis, Adv. Mater., 2009, 21, 325-328.

8 J. M. Spurgeon, S. W. Boettcher, M. D. Kelzenberg, B. S. Brunschwig, H. A. Atwater and N. S. Lewis, Adv. Mater., 2010, 22, 3277-3281.

9 H. Cui, S. Li, S. Deng, H. Chen and C. Wang, ACS Sens., 2017, 2, 386-393.
10 X. Chen, J. Wen, J. Zhou, Z. Zheng, D. An, H. Wang, W. Xie, R. Zhan, N. Xu, J. Chen, J. She, H. Chen and S. Deng, J. Opt., 2018, 20, 024012.

11 K. Kneipp, Y. Wang, H. Kneipp, L. T. Perelman, I. Itzkan, R. R. Dasari and M. S. Feld, Phys. Rev. Lett., 1997, 78, 1667.

12 B. Sharma, R. R. Frontiera, A. I. Henry, E. Ringe and R. P. V. Duyne, Mater. Today, 2012, 15, 16-25.

13 F. M. Liu and M. Green, J. Mater. Chem., 2004, 14, 1526-1532. 14 P. Hu, X. S. Zheng, C. Zong, M. H. Li, L. Y. Zhang, W. Li and B. Ren, J. Raman Spectrosc., 2014, 45, 565-573.

15 Y. Sun and G. P. Wiederrecht, Small, 2007, 3, 1964-1975.

16 Y. J. Liu, H. Y. Chu and Y. P. Zhao, J. Phys. Chem. C, 2010, 114, 8176-8183.

17 R. Ji, W. Sun and Y. Chu, RSC Adv., 2014, 4, 6055-6059.

18 X. Li and P. W. Bohn, Appl. Phys. Lett., 2000, 77, 2572-2574. 19 J. Y. Li, C. H. Hung and C. Y. Chen, Sci. Rep., 2017, 7, 17177. 20 Y. Chen, L. Li, C. Zhang, C. C. Tuan, X. Chen, J. Gao, Y. Ding and C. P. Wong, Nano Lett., 2017, 17, 1014-1019.

21 C. Y. Chen, T. C. Wei, P. H. Hsiao and C. H. Hung, ACS Appl. Energy Mater., 2019, 2, 4873-4881.

22 O. J. Hildreth, D. Brown and C. P. Wong, Adv. Funct. Mater., 2011, 23, 3119-3128.

23 I. R. Putra, J. Y. Li and C. Y. Chen, Appl. Surf. Sci., 2019, 478, 725-732.

24 C. Chartier, S. Bastide and C. L. Clement, Electrochim. Acta, 2008, 53, 5509-5516.

25 Z. Huang, X. Zhang, M. Reiche, L. Liu, W. Lee, T. Shimizu, S. Senz and U. Gosele, Nano Lett., 2008, 8, 3046-3051.

26 W. Chern, K. Hsu, I. S. Chun, B. P. de Azeredo, N. Ahmed, K. H. Kim, J. min Zuo, N. Fang, P. Ferreira and X. Li, Nano Lett., 2010, 10, 1582-1588.

27 C. Y. Chen, P. H. Hsiao, T. C. Wei, T. C. Chen and C. H. Tang, Phys. Chem. Chem. Phys., 2017, 19, 11786-11792.

28 Z. Huang, N. Geyer, P. Werner, J. Boor and U. Gosele, Adv. Mater., 2011, 23, 285-308.

29 X. Zhong, Y. Qu, Y. C. Lin, L. Liao and X. Duan, ACS Appl. Mater. Interfaces, 2011, 3, 261-270.

30 S. Li, W. Ma, Y. Zhou, X. Chen, Y. Xiao, M. Ma, W. Zhu and F. Wei, Nanoscale Res. Lett., 2014, 9, 196.

31 S. L. Kleinman, R. R. Frontiera, A. I. Henry, J. A. Dieringer and R. P. V. Duyne, Phys. Chem. Chem. Phys., 2013, 15, 21-36.

32 S. M. Stranahan and K. A. Willets, Nano Lett., 2010, 10, 37773784.

33 C. Farcau and S. Astilean, J. Phys. Chem. C, 2010, 114, 1171711722.

34 J. M. McLellan, Z. Y. Li, A. R. Siekkinen and Y. Xia, Nano Lett., 2007, 7, 1013-1017.

35 X. Chen, C. H. Cui, Z. Guo, J. H. Liu, X. J. Huang and S. H. Yu, Small, 2011, 7, 858-863.

36 Y. Wang, H. Chen, S. Dong and E. Wang, J. Raman Spectrosc., 2007, 38, 515-521. 